\title{
Evaluación del segmento anterior y cambios en la presión intraocular después de la cirugía de catarata por facoemulsificación
}

\section{Assessment of the anterior segment and intraocular pressure changes after phacoemulsification}

\author{
Adem Ugurlu* y Nurdan Gamze-Tasli \\ Departamento de Oftalmología, Facultad de Medicina, Erzincan Binali Yildirim University, Erzincan, Turquía
}

\begin{abstract}
Resumen
Objetivo: Investigar los cambios en los parámetros del segmento anterior y los valores de presión intraocular después de una cirugía de facoemulsificación sin complicaciones. Métodos: Se incluyeron en el estudio un total de 118 pacientes que se sometieron a cirugía de catarata por facoemulsificación sin incidentes con implantación de lente intraocular. Los pacientes se sometieron a un examen oftalmológico detallado que incluyó agudeza visual mejor corregida, biomicroscopia con lámpara de hendidura, gonioscopia, fundoscopia, medición de la presión intraocular (PIO) y evaluación del segmento anterior en la basal (preoperatoriamente), la primera semana, los meses primero, tercero y sexto, y el primer año después de la cirugía. Resultados: Cincuenta y nueve pacientes eran hombres y cincuenta y nueve mujeres. La edad media de los pacientes fue de $64.9 \pm 10.8$ (25-89) años. La PIO media fue de $16.9 \pm 1.9$ (11-20) mm Hg en la basal y de $12.9 \pm 1.8$ $(10-18) \mathrm{mm} \mathrm{Hg}$ el primer año después de la cirugía $(p<0.001)$. La profundidad media de la cámara anterior fue de $2.50 \pm$ $0.35(1.71-3.45) \mathrm{mm}$ en la basal y de $3.98 \pm 0.40(2.98-4.60) \mathrm{mm}$ al primer año postoperatorio $(p<0.001)$. El ángulo medio de la cámara anterior fue de $33.06 \pm 3.91$ (29.7-45) grados en la basal del estudio y de $38.6 \pm 4.8$ (32.2-50.2) grados al primer año postoperatorio $(p<0.001)$. Conclusiones: Este estudio mostró que la PIO disminuyó, pero otros hallazgos, como la profundidad de la cámara anterior, el ángulo de la cámara anterior y el volumen de la cámara anterior, aumentaron en el seguimiento a corto y largo plazo después de la cirugía de facoemulsificación.
\end{abstract}

Palabras clave: Evaluación. Segmento anterior. Presión intraocular. Facoemulsificación.

\section{Abstract}

Aim: To investigate the changes in anterior segment parameters and intraocular pressure after uneventful phacoemulsification. Methods: $A$ total of 118 patients who underwent uneventful phacoemulsification and intraocular lens (IOL) implantation were included in the study. The patients underwent a detailed ophthalmologic examination including best-corrected visual acuity (BCVA), slit-lamp biomicroscopy, gonioscopy, fundoscopy, intraocular pressure (IOP) measurement, and anterior segment evaluation at baseline (preoperatively) and one week, one, three and six months, as well as one year after surgery. Results: Fifty-nine patients were male and fifty-nine were female. Mean age was $64.9 \pm 10.8$ (25-89) years. Mean IOP at baseline was $16.9 \pm 1.9(11-20) \mathrm{mm} \mathrm{Hg}$. Mean IOP was $12.9 \pm 1.8(10-18) \mathrm{mm} \mathrm{Hg}$ one year after surgery $(P<0.001)$. Mean

Correspondencia:

*Adem Ugurlu

Basbaglar Mh. 1429 Sk 2/1

Fecha de recepción: 24-04-2020

Fecha de aceptación: 25-06-2020

Correo electrónico: ademugurlu88@ hotmail.com DOI: 10.24875/RMO.M20000132
Disponible en internet: 09-11-2020 Rev Mex Oftalmol. 2020;94(6):252-259

www.rmo.com.mx 0187-4519/๑ 2020 Sociedad Mexicana de Oftalmología. Publicado por Permanyer. Este es un artículo open access bajo la licencia CC BY-NC-ND (http://creativecommons.org/licenses/by-nc-nd/4.0/). 
anterior chamber depth $(A C D)$ was $2.50 \pm 0.35$ (1.71-3.45) $\mathrm{mm}$ at baseline and $3.98 \pm 0.40$ (2.98-4.60) $\mathrm{mm}$ one year after surgery $(P<0.001)$. Mean anterior chamber angle $(A C A)$ was $33.06 \pm 3.91(29.7-45)$ degrees at baseline and $38.6 \pm 4.8$ (32.2-50.2) degrees one year after surgery $(P<0.001)$. Conclusions: This study showed that an IOP decrease. Other findings, such as anterior chamber depth, anterior chamber angle, and anterior chamber volume (ACV) increased in the short- and long-term follow-up after phacoemulsification.

Key words: Assessment. Anterior segment. Intraocular pressure. Phacoemulsification.

\section{Antecedentes}

Una catarata es una condición caracterizada por la opacificación y la pérdida de transparencia del cristalino. Hay varias razones para el desarrollo de cataratas..$^{1-3}$ Las cataratas a menudo son adquiridas y rara vez son congénitas. ${ }^{4-6}$ El tratamiento de las cataratas es la cirugía, y la facoemulsificación es el método quirúrgico más utilizado para tratar este trastorno ocular. ${ }^{6-8} \mathrm{La}$ disminución de la agudeza visual es una de las principales indicaciones para la facoemulsificación. 2,6,7

La facoemulsificación con implante de lentes intraoculares (LIO) pueden causar alteraciones de los parámetros intraoculares. En particular, ocasionan un aumento de la profundidad de la cámara anterior (PCA), el ángulo de la cámara anterior (ACA) y el volumen de la cámara anterior (VCA). ${ }^{9-15} \mathrm{El}$ engrosamiento del cristalino relacionado con la edad induce muchas alteraciones estructurales relacionadas con la vía de salida del humor acuoso que pueden conducir a un aumento de la presión intraocular (PIO), asociada con glaucoma. Muchos estudios han investigado los mecanismos de reducción de la PIO después de la facoemulsificación midiendo los cambios postoperatorios de los parámetros biométricos del segmento anterior para mantener el acceso al flujo de salida de acuoso..$^{16-18}$

La cirugía moderna de catarata produce una reducción de la PIO., 8,9,16-19 Como resultado de la extracción del cristalino y la implantación de un LIO, que es más delgado que el cristalino, el volumen y la profundidad de la cámara anterior aumentan, el ángulo iridocorneal se ensancha y el iris se mueve hacia atrás. ${ }^{20} \mathrm{Al}$ mismo tiempo, la secreción endógena de prostaglandina F2, un mediador inflamatorio, se eleva debido al trauma quirúrgico, lo que provoca un aumento del flujo de humor acuoso por vía uveoescleral. ${ }^{21}$ Aunque existe una investigación sustancial sobre los cambios a corto plazo de la PIO y los parámetros del segmento anterior después de una cirugía de catarata sin incidentes, no hay muchos estudios clínicos que evalúen los cambios a largo plazo. Este estudio prospectivo es uno de los pocos estudios en los que se evaluaron tanto la PIO como los parámetros detallados del segmento anterior antes y después de la facoemulsificación durante un período de un año.

En esta investigación prospectiva, las alteraciones de los parámetros del segmento anterior y los valores de la PIO se investigaron después de una facoemulsificación sin incidentes durante un período de seguimiento de un año.

\section{Métodos}

Entre noviembre de 2017 y mayo de 2018, se incluyeron en el estudio 118 ojos de 118 pacientes que se sometieron a facoemulsificación sin complicaciones e implantación de un lente intraocular plegables, con diagnóstico de catarata en la Clínica de Oftalmología de la Facultad de Medicina de la Universidad Erzincan Binali Yildirim. El estudio se realizó de acuerdo con los principios de la Declaración de Helsinki, y se obtuvo el consentimiento informado de cada paciente antes del estudio. El Comité de Ética Institucional de la Universidad Erzincan Binali Yildirim también aprobó el estudio. (Número de decisión del comité de ética: 33216249-804.01-E.13165).

\section{Evaluación clínica}

Los pacientes se sometieron a un examen ocular detallado que incluyó la agudeza visual mejor corregida (AVMC), biomicroscopia con lámpara de hendidura, gonioscopia, fundoscopia, medición de PIO y evaluación del segmento anterior en la basal (preoperatoriamente) y una semana, uno, tres y seis meses, así como un año después de la facoemulsificación. La medición de la PIO se repitió cinco veces al día (a las 9:00, 11:00, 13:00, 15:00, 18:00), un día antes de la cirugía. Se calculó el valor medio de la PIO y se anotó como el valor basal (preoperatorio) de la PIO. También se realizó una evaluación del ángulo utilizando el sistema de Schaffer, y ninguno de los pacientes tenía grado 102 por gonioscopia.

La agudeza visual se midió en condiciones fotópicas utilizando cartillas de Snellen con la unidad oftálmica Nidek (Nidek Co., Ltd., Japón). El examen biomicroscópico con lámpara de hendidura se realizó utilizando la unidad 
Tabla 1. Cambios de la presión intraocular y de los parámetros del segmento anterior antes y después de la facoemulsificación

\begin{tabular}{|l|c|c|c|c|c|c|c|}
\hline & $\begin{array}{c}\text { Antes de la } \\
\text { cirugía }\end{array}$ & $\begin{array}{c}1 \text { semana } \\
\text { después de la cirugía }\end{array}$ & $\begin{array}{c}1 \text { mes después } \\
\text { de la cirugía }\end{array}$ & $\begin{array}{c}3 \text { meses después } \\
\text { de la cirugía }\end{array}$ & $\begin{array}{c}6 \text { meses después } \\
\text { de la cirugía }\end{array}$ & $\begin{array}{c}1 \text { año } \\
\text { después de la cirugía }\end{array}$ \\
$\begin{array}{l}\text { Valor } \\
\text { de p }\end{array}$ \\
\hline PIO & $16.9 \pm 1.9$ & $16.5 \pm 1.6$ & $13.9 \pm 1.7$ & $12.5 \pm 1.7$ & $12.7 \pm 1.6$ & $12.9 \pm 1.8$ & $<0.001$ \\
\hline PCA & $2.50 \pm 0.35$ & $3.96 \pm 0.39$ & $4.08 \pm 0.36$ & $4.11 \pm 0.34$ & $3.99 \pm 0.41$ & $3.98 \pm 0.40$ & $<0.001$ \\
\hline ACA & $33.06 \pm 3.91$ & $40.00 \pm 5.55$ & $40.16 \pm 5.43$ & $40.17 \pm 5.28$ & $39.1 \pm 4.92$ & $38.61 \pm 4.84$ & $<0.001$ \\
\hline VCA & $129.44 \pm 29.29$ & $173.61 \pm 25.78$ & $177.81 \pm 24.55$ & $179.21 \pm 23.90$ & $175.15 \pm 24.06$ & $174.70 \pm 26.01$ & $<0.001$ \\
\hline GCC & $548.78 \pm 42.13$ & $581.32 \pm 47.92$ & $556.98 \pm 41.92$ & $553.88 \pm 41.49$ & $550.81 \pm 42.19$ & $549.37 \pm 41.36$ & $<0.001$ \\
\hline
\end{tabular}

PIO: Presión intraocular; PCA: Profundidad de la cámara anterior; ACA: Ángulo de la cámara anterior; VCA: Volumen de la cámara anterior; GCC: Grosor corneal central.

oftálmica Nidek (Nidek Co., Ltd., Japón). El examen gonioscópico se realizó con una lente gonioscópica de tres espejos Volk G-3 (Volk Optical, Inc., EE. UU.). El examen fundoscópico se realizó con lentes de 90 D Volk (Volk Optical, Inc., EE. UU.) bajo biomicroscopia. Las mediciones de PIO se realizaron con un tonómetro de aplanamiento Goldmann (Haag-Streit, Reino Unido). Todos los exámenes oculares fueron realizados por el mismo clínico para evitar la variación entre observadores.

La profundidad, el volumen y el ángulo de la cámara anterior, el grosor corneal central (GCC) y la longitud axial se midieron con un topógrafo OPD scan-3 (Nidek Co., Ltd., Japón) en todos los pacientes antes de la cirugía. Las mediciones fueron repetidas una semana, uno, tres, seis y doce meses después de la cirugía por el mismo miembro del personal para evitar la variación entre observadores.

En todos los casos, el mismo cirujano experimentado realizó una incisión corneal clara de 2,4 mm, utilizando el Infiniti vision system (Alcon, Inc.) con técnicas de facoemulsificación 'stop and chop' o 'chip and flip' en todos los pacientes. En todos los casos, la facoemulsificación se completó con éxito con la colocación de un LIO AcrySof IQ (Alcon Laboratories, Inc.) en la bolsa capsular. Las incisiones se hidrataron con solución salina balanceada después de retirar el viscoelástico (DisCo-Visc Alcon Laboratories, Inc., Fort Worth, TX, EE. UU.). A todos los pacientes se les recetó un antibiótico tópico y gotas oculares con esteroides cuatro veces al día durante cuatro semanas después de la cirugía.

\section{Criterios de exclusión}

Los pacientes con complicaciones durante la cirugía de facoemulsificación, o aquellos con antecedentes de enfermedad corneal, pseudoexfoliación, ángulo estrecho, glaucoma, uveítis, cirugía ocular previa o trauma, así como trastornos del segmento posterior, fueron excluidos del estudio. También se excluyeron los pacientes con inflamación prolongada de la cámara anterior después de la facoemulsificación y aquellos que necesitaron utilizar gotas oculares con esteroides después de un mes de la cirugía.

\section{Análisis estadístico}

Se utilizó el Statistical Package for the Social Sciences versión 22.0 (SPSS Inc., Chicago, IL, EE. UU.) para los análisis estadísticos, y un valor de $p<.05$ se consideró significativo con un intervalo de confianza (IC) del 95\%. Todos los datos se expresaron como media, desviación estándar, valores mínimo y máximo. La prueba de chi cuadrada se utilizó para comparar datos nominales. Las pruebas de Pearson o Spearman se utilizaron para el análisis de correlación. La distribución de los datos se examinó mediante la prueba de Kolmogorov-Smirnov. Se utilizó un ANOVA o prueba $t$ para comparar los datos distribuidos normalmente, mientras que la prueba $U$ de Mann Whitney y la prueba de Kruskal Wallis se utilizaron para comparar los datos sin distribución normal. El análisis de varianza de Friedman y la prueba de Wilcoxon se utilizaron para comparar mediciones recurrentes.

\section{Resultados}

En esta investigación, 59 pacientes fueron hombres y 59 mujeres. La edad media de los participantes fue de $64.9 \pm 10.825-89]$ años. La longitud axial promedio del globo ocular fue de $23.5 \pm 0.921 .29-25.66] \mathrm{mm}$. Los cambios en la PIO antes y después de la cirugía se muestran en la Tabla 1 y la Figura 1. 


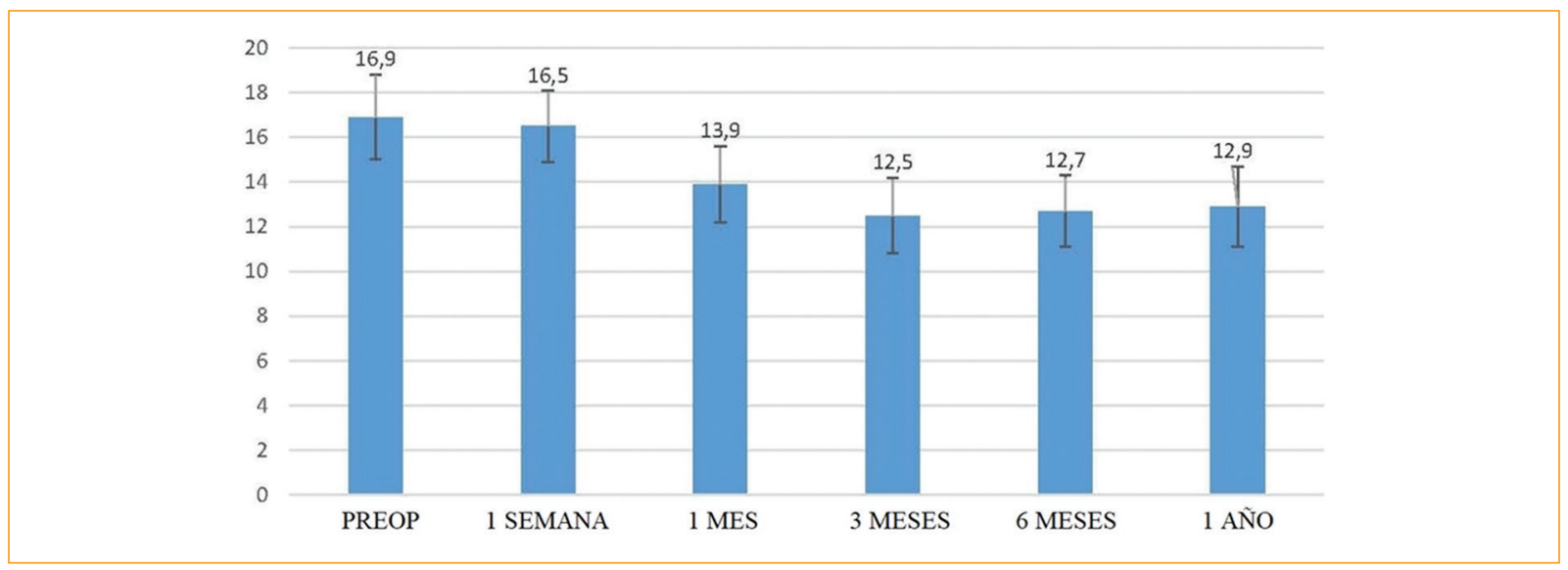

Figura 1. Cambios de presión intraocular (PIO) durante el seguimiento.

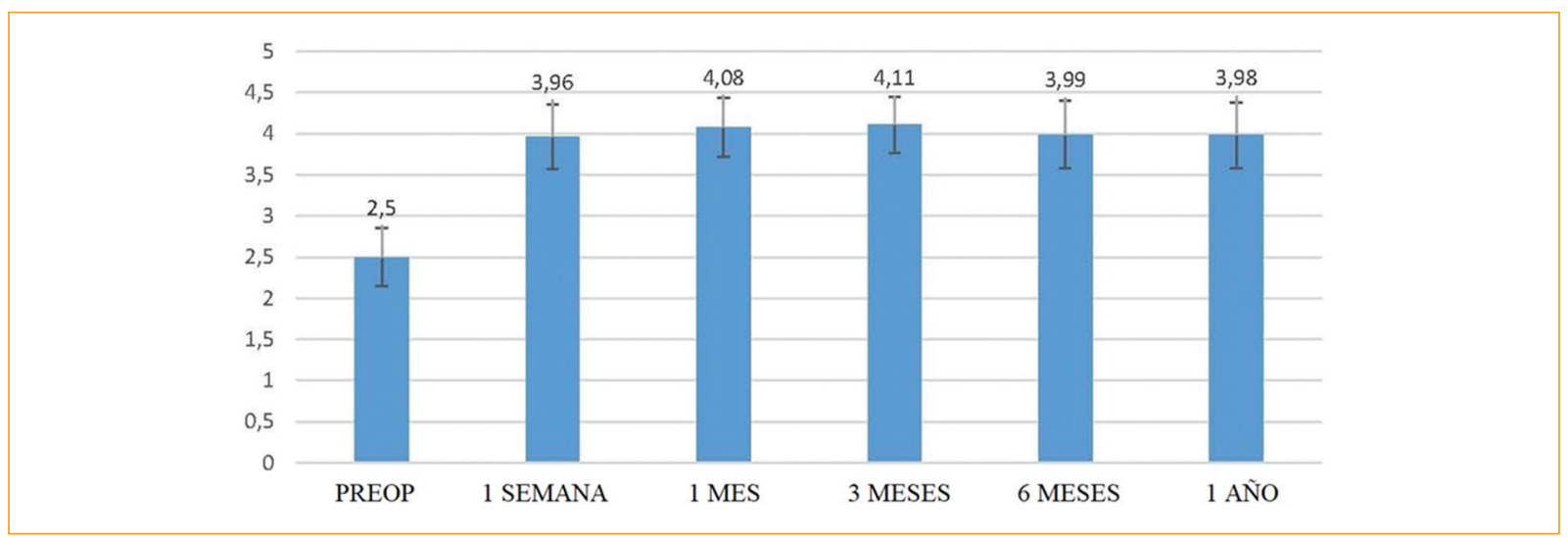

Figura 2. Cambios de profundidad de la cámara anterior (PCA) durante el seguimiento.

No hubo diferencias estadísticamente significativas entre el valor basal y la medición postoperatoria de 1 semana cuando se compararon los valores de PIO $(P=0.708)$ (IC 95\%: -0.948 a +1.722). Sin embargo, se observó una diferencia estadísticamente significativa entre los valores basales y los valores de PIO postoperatorios a los $1,3,6$ y 12 meses $(P<.001)$ (IC 95\%: $0.618-1.422,1.957-2.818,1.681-2.605$ y $1.497-2.401$, respectivamente). También hubo una diferencia estadísticamente significativa entre los valores de PIO postoperatorios de la primera semana y los valores a los $1,3,6$ y 12 meses $(P<.001)$ (IC 95\%: 0.225-0.978, 1.561-2.378, 1.382-2.144, y 1.092-1.969, respectivamente). Del mismo modo, se observó una diferencia significativa entre las mediciones de PIO del primer mes y las de los 3 y 6 meses $(P<.001)$ (IC 95\%: 0.937-1.798 y 0.685-1.627, respectivamente). Las mediciones postoperatorias de la PIO del primer mes y e primer año también difirieron significativamente ( $P=.018$ ) (IC 95\%: 0.428-1.231). Sin embargo, no hubo diferencias significativas en los valores de PIO a los 3 meses en comparación con los 6 meses y un año después de la facoemulsificación $(P>.05)$ (IC 95\%: -0.803 a -0.375 y -0.908 a -0.456 , respectivamente). La diferencia en la PIO tampoco fue significativa al comparar los puntos de tiempo de 6 meses y 1 año ( $P>$.05) (IC 95\%: -0.112 a +0.024).

Las alteraciones de la PCA antes y después de la facoemulsificación, se muestran en la Tabla 1 y la Figura 2. Hubo una diferencia estadísticamente significativa en las mediciones promedio de PCA entre la basal y los valores postoperatorios de la semana 1 , mes 1 , mes 3, mes 6 y 1 año $(P<.001)$ (IC 95\%: -1.583 a $-1.328,-1.693$ a $-1.459,-1.723$ a $-1.487,-1.653$ a -1.391 y -1.603 a -1.353 , respectivamente). También hubo una diferencia significativa entre las mediciones de PCA de la semana 1 y el mes $1(P=.048)($ IC 95\%: -0.197 


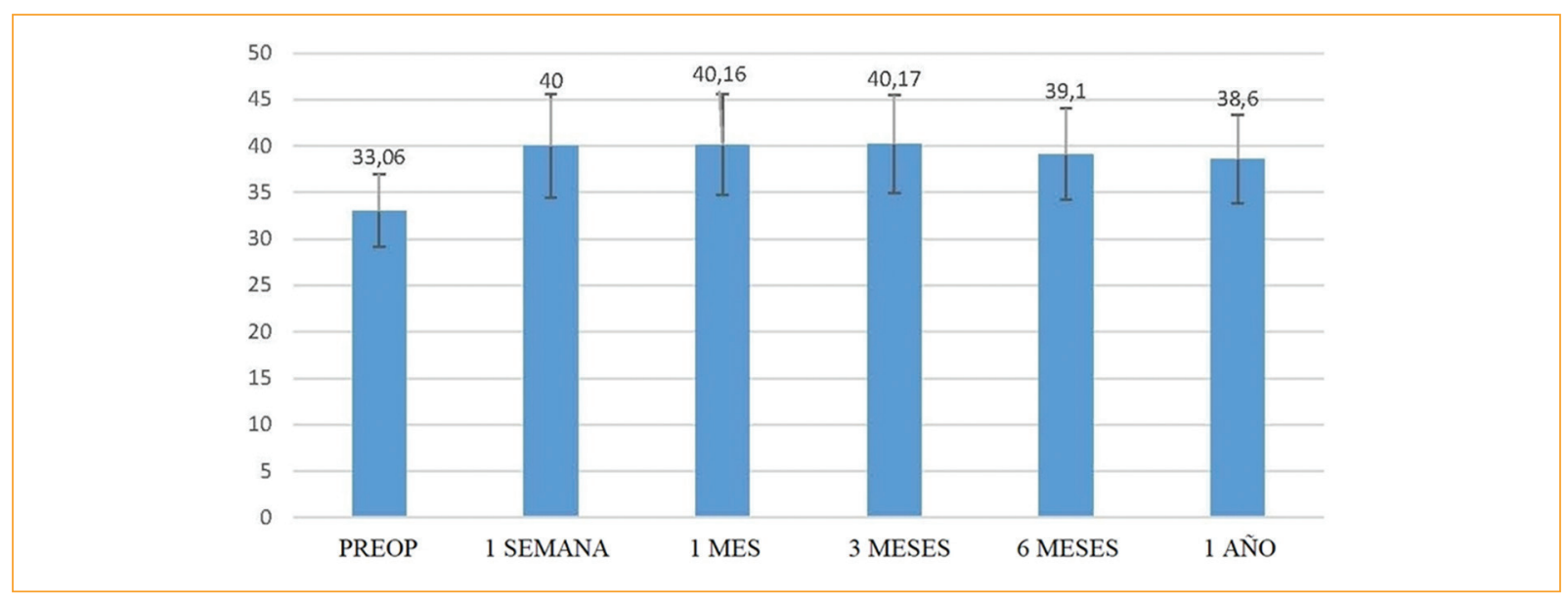

Figura 3. Cambios del ángulo de la cámara anterior (ACA) durante el seguimiento.

a -0.044), así como en las dimensiones de la PCA medidas en la primera semana en comparación con el tercer mes postoperatorio ( $P=.003$ ) (IC 95\%: -0.238 a -0.061). Sin embargo, no se observaron diferencias estadísticamente significativas de las mediciones de PCA entre la semana 1 y el mes 6 y el año 1 después de la facoemulsificación $P=.494$ ) (IC 95\%: -0.119 a +0.044 y -0.108 a +0.065 , respectivamente). Del mismo modo, las dimensiones postoperatorias de PCA del primer y tercer mes no difirieron significativamente $(P>$.05) (IC 95\%: -0.085 a +0.026). La diferencia entre las mediciones de PCA del primer mes y del primer año después de la cirugía, fue estadísticamente significativa $(P<.001)$ (IC 95\%: 0.026 - 0.170). Se observó otra diferencia estadísticamente significativa entre las dimensiones de PCA postoperatorias del mes 3 en comparación con el mes 6 y 1 año $(P<.001)$ (IC 95\%: $0.011-0.198$ y $0.069-0.185)$. Sin embargo, no hubo una diferencia significativa entre las mediciones de PCA del mes 6 y del año 1 después de la cirugía $(P>$ .05) (IC 95\%: -0.188 a +0.074).

Las alteraciones del ACA antes y después de la cirugía se presentan en la Tabla 1 y la Figura 3. Hubo una diferencia significativa entre los valores de ACA basales y postoperatorios de la primera semana, primer mes, tercer mes, sexto mes y primer año $(P<.001)$. (IC 95\%: -12.545 a -9.330, -12.784 a -9.410, -12.812 a $-9.400,-12.411$ a -8.847 y -11.033 a -8.067 , respectivamente). No hubo diferencias significativas entre la semana 1 postoperatoria y 1 y 3 meses postcirugía en términos del ACA ( $P>.05$ ) (IC 95\%: -1.062 a +0.744 y -1.176 to +0.839 , respectivamente). Se observó una diferencia significativa en este parámetro cuando la medición de la semana 1 se comparó con las mediciones de 6 meses y 1 año $(P=.001)$ (IC 95\%: $0.359-1.839$ y $0.451-2.324$, respectivamente). También hubo una diferencia significativa entre los valores de 1 mes y 6 meses y 1 año después de la cirugía $(P<.001)$ (IC 95\%: 0.592- 1.998 y $0.704-2.390$, respectivamente), así como entre el mes 3 y el mes 6 y el año $1(P<.001)$ (IC $95 \%$ : $0.764-2.248$ y $0.805-2.307$, respectivamente). Sin embargo, no se observaron diferencias significativas en los valores de ACA entre el mes 6 y el año $1(P>$.05) (IC 95\%: -0.485 a +1.062).

Los cambios del VCA antes y después de la cirugía se presentan en la Tabla 1 y la Figura 4. Hubo una diferencia estadísticamente significativa entre las mediciones de VCA basales y postoperatorias de la primera semana, primer mes, tercer mes, sexto mes y primer año $(P<.001)$ (IC 95\% -50.046 a -38.301, -54.320 a -42.415, -55.664 a $-43.887,-53.844$ a -41.427 y -51.631 a -38.899). También hubo una diferencia significativa entre las mediciones medias postoperatorias de la primera semana y las mediciones del primer y tercer mes $(P=.002$ y $P<.001$, respectivamente) (IC 95\%: -7.504 a -0.883 y -8.601 a -2.603 , respectivamente). Sin embargo, no se observaron diferencias significativas entre las mediciones de la semana 1 y 6 meses y 1 año después de la cirugía $(P>.05)$ (IC 95\%: -3.876 a +1.145 y -4.701 a +2.517, respectivamente). Tampoco hubo diferencias significativas entre los valores de VCA del primer y tercer mes $(P=.608)$ (IC 95\%: -3.239 a +0.423). Se observó un diferencia significativa entre las mediciones de VCA postoperatorias del mes 1 en comparación con el mes 6 y el año $1(P=.001)$ (IC 95\%: $0.626-4.497$ y 0.815 5.389 , respectivamente), así como entre el tercer mes y 


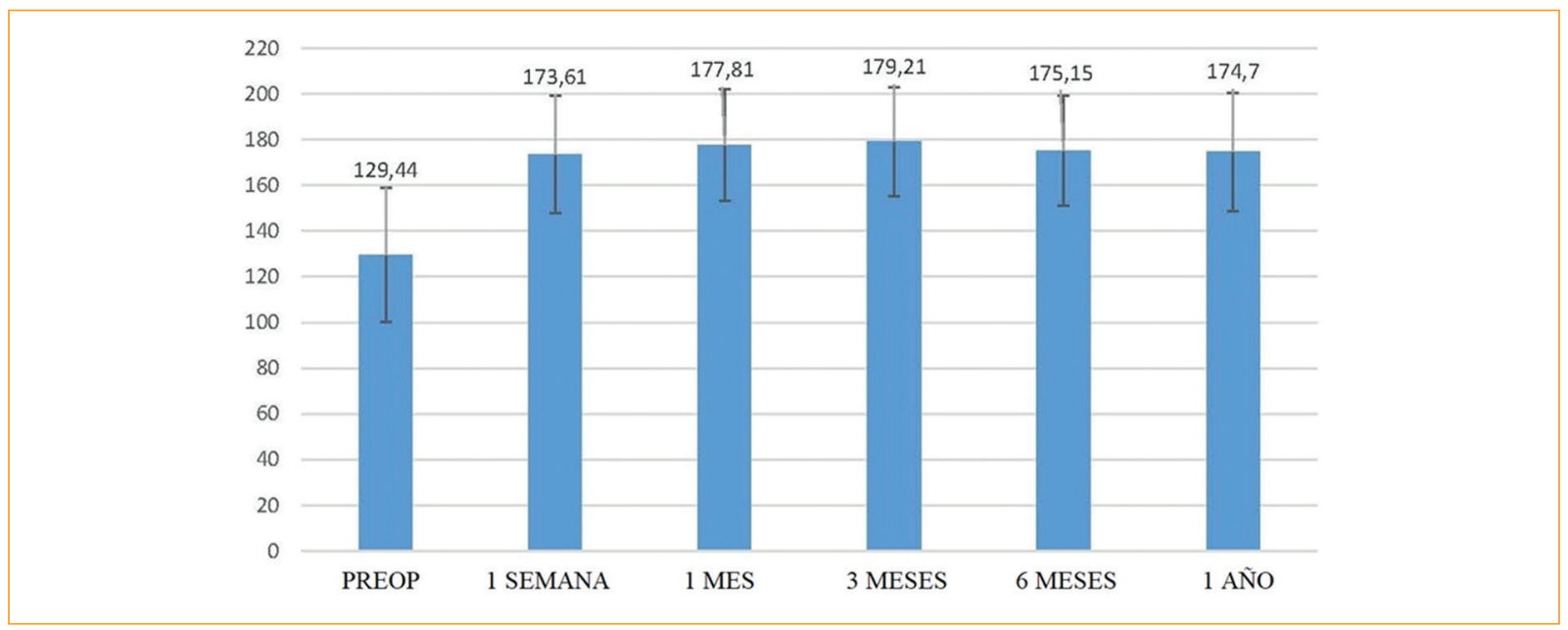

Figura 4. Cambios del volumen de la cámara anterior (VCA) durante el seguimiento.

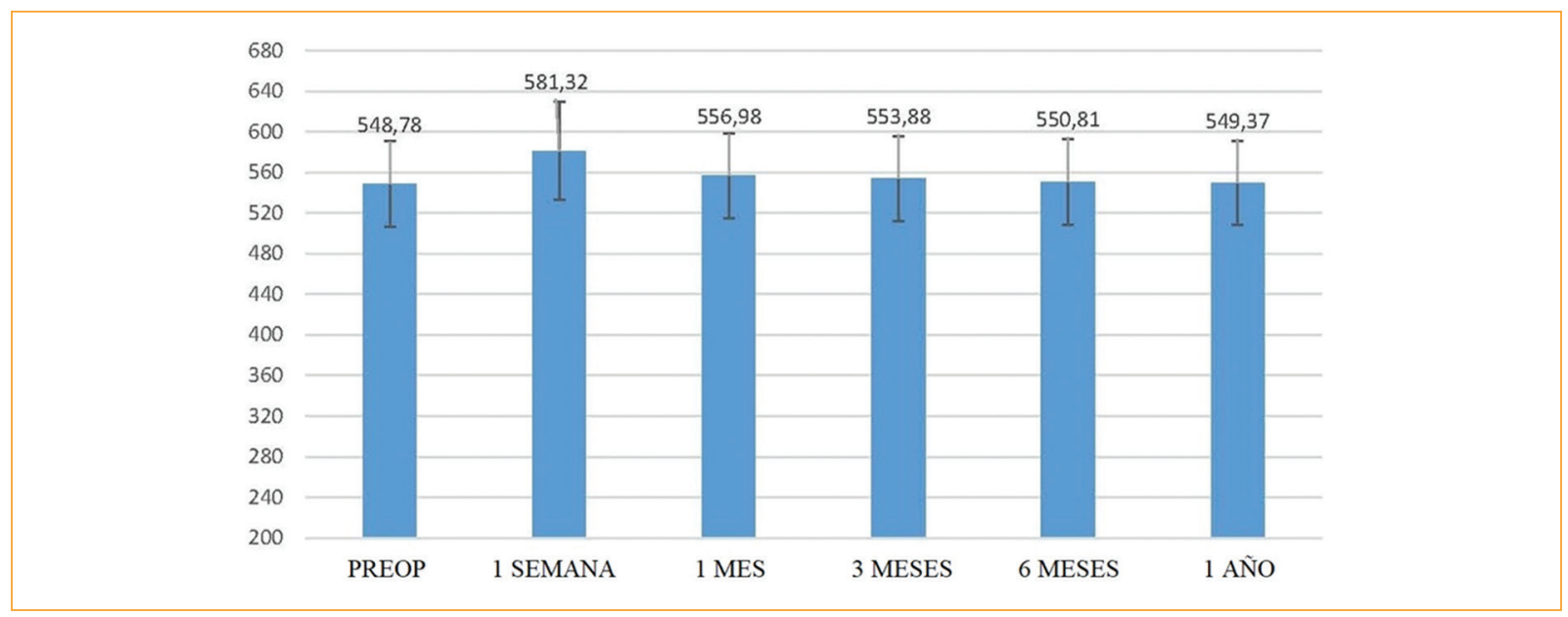

Figura 5. Cambios del grosor corneal central (GCC) durante el seguimiento.

el sexto mes postoperatorios y el primer año $(P<.001)$ (IC 95\%: $1.867-5.979$ y $2.493-6.528$, respectivamente). No hubo diferencias significativas entre los valores de VCA postoperatorios en las mediciones del mes 6 y del año $1(P>.05)$ (IC 95\%: -2.859 a +4.864).

Las alteraciones del GCC antes y después de la cirugía se muestran en la Tabla 1 y la Figura 5. Hubo una diferencia estadísticamente significativa entre las mediciones de GCC basales y postoperatorias de la primera semana, primer mes y tercer mes $(P<.001)($ IC 95\% -39.247 a $-25.834,-11.878$ a -4.530 y -8.971 a -1.233 , respectivamente). No se observaron diferencias estadísticamente significativas entre las mediciones basales y postoperatorias de GCC a los 6 meses y un año $(P>.05)$ (IC 95\%: -6.578 a +0.547 y -4.343 a +3.159 , respectivamente). Se observó una diferencia significativa entre las mediciones basales y de la primera semana, primer mes, tercer mes, sexto mes y primer año $(P<.001)$. (IC 95\%: $18.430-$ $30.243,21.552-33.326,23.674-36.778$ у $25.783-$ 38.115 , respectivamente). Sin embargo, no se observaron diferencias significativas entre los valores de 1 semana y 6 meses y 1 año después de la cirugía $(P>.05)$ (IC 95\%: $-2.774 \mathrm{a}+3.684)$. El GCC medido en el primer mes postoperatorio difirió significativamente en comparación con las mediciones postoperatorias del sexto mes y el primer año $(P<.001)$ (IC 95\%: 2.498 - 7.978 y $4.864-10.361$, respectivamente). También hubo una diferencia significativa cuando se comparó el valor de GCC postoperatorio del tercer mes con los valores del sexto mes y del primer año $(P<.001)($ IC 95\%: $1.984-4.763$ y $2.542-6.478$, 
respectivamente). Sin embargo, la diferencia entre los valores postoperatorios del sexto mes y del primer año no fue significativa $(P>.05)$ (IC 95\%: $-3.084 \mathrm{a}+4.258)$.

\section{Discusión}

Este estudio tuvo como objetivo evaluar los efectos de la facoemulsificación en la PIO y los parámetros del segmento anterior. En la literatura, Yang et al. examinaron los cambios de PIO después de la cirugía de catarata, y encontraron que los niveles de PIO disminuyeron significativamente después de un seguimiento de tres meses. ${ }^{22}$ En nuestro estudio, no hubo diferencias estadísticamente significativas entre las mediciones basales y postoperatorias de la PIO de la primera semana en los pacientes incluidos en el estudio. Sin embargo, los valores medios de la PIO fueron significativamente más bajos un mes después de la cirugía en comparación con los valores preoperatorios y postoperatorios de la primera semana. Kashiwagi et al. mostraron que los valores de la PIO disminuyen después de la cirugía, y que la reducción de la PIO es significativamente mayor en los ojos con una PCA preoperatoria estrecha. ${ }^{23}$

Chen et al., investigaron las alteraciones de la PCA después de la facoemulsificación, e informaron que las mediciones de PCA posoperatorias del primer día, la primera semana y el primer mes, fueron significativamente más altas en comparación con las del período preoperatorio, y que la PCA comenzó a estabilizarse en la visita de la primera semana después de la cirugía. ${ }^{24}$ En nuestro estudio prospectivo, la PCA continuó aumentando hasta tres meses después de la cirugía, y se observó que se estabilizó seis meses después de la cirugía.

Lee et al. examinaron los parámetros biométricos del segmento anterior después de la cirugía de catarata, y encontraron que el ACA al tercer mes de seguimiento aumentó significativamente en comparación con el valor basal..$^{25}$ Altan et al. evaluaron los cambios de PCA, PIO y ACA después de una facoemulsificación sin incidentes e informaron resultados similares, como valores reducidos de PIO y el aumento de PCA y $\mathrm{ACA} .^{26} \mathrm{En}$ nuestro estudio, el ACA aumentó después de la cirugía de cataratas y se mantuvo estable entre una semana y tres meses después de la cirugía. Los valores de ACA disminuyeron ligeramente seis meses después de la cirugía, pero seguían siendo mayores en comparación con los valores preoperatorios. Observamos que los valores de ACA se estabilizaron seis meses después de la cirugía de catarata.
Con respecto a las mediciones de VCA, el valor de la primera semana fue significativamente mayor que el valor basal. El aumento de VCA continuó en la visita del primer mes después de la cirugía. Sin embargo, los valores medios de VCA en el sexto mes y el primer año después de la cirugía, casi retornaron a los niveles de la semana 1. Por otro lado, las mediciones postoperatorias de VCA en el sexto mes y primer año, fueron significativamente más altas en comparación con el valor basal medio, y significativamente más bajas en comparación con las mediciones postoperatorias del primer y el tercer mes.

Cetinkaya et al. evaluaron el efecto de la cirugía de facoemulsificación en la PIO y la anatomía del segmento anterior, y encontraron que los valores de PCA, PIO y GCC retornaron a los niveles preoperatorios durante un seguimiento a largo plazo después de la cirugía. ${ }^{14}$ En nuestro estudio, los valores de GCC también disminuyeron a los niveles preoperatorios en las visitas del sexto mes y el primer año después de la cirugía. Este estudio mostró que el GCC se estabilizó 6 meses después de la cirugía.

\section{Conclusión}

Este estudio mostró que después de la facoemulsificación, la PIO disminuye en el seguimiento a corto y largo plazo en comparación con el período preoperatorio. Sin embargo, parámetros como la profundidad, el ángulo y el volumen de la cámara anterior aumentan en el seguimiento a corto y largo plazo después de la cirugía. Aunque el GCC aumentó en el postoperatorio temprano, volvió a los niveles preoperatorios a largo plazo.

\section{Conflicto de intereses}

Este estudio no fue financiado. De acuerdo con la obligación ética como investigadores, todos los autores declaran que no tienen ningún conflicto de intereses con respecto a esta investigación.

\section{Responsabilidades éticas}

Protección de personas y animales. Los autores declaran que para esta investigación no se han realizado experimentos en seres humanos ni en animales.

Confidencialidad de los datos. Los autores declaran que en este artículo no aparecen datos de pacientes. 


\section{Derecho a la privacidad y consentimiento informado. Los autores declaran que en este artículo no aparecen datos de pacientes.}

\section{Referencias}

1. Kanski JJ, Bowling B. Lens. In: Nischal KK, Pearson A. (editor) Clinical Ophtalmology A Systematic Approach. 7th ed. Edinburgh: Elsevier/Saunders; 2011;163-191.

2. Thompson J, Lakhani N. Cataracts. Prim Care. 2015;42(3):409-23.

3. Lee CM, Afshari NA. The global state of cataract blindness. Curr Opin Ophthalmol. 2017;28(1):98-103.

4. Chan WH, Biswas S, Ashworth JL, Lloyd IC. Congenital and infantile cataract: aetiology and management. Eur J Pediatr. 2012;171(4):625-30.

5. Lin H, Lin D, Liu Z, Long E, Wu X, Cao Q, et al. Novel Congenital Catarac Category System Based on Lens Opacity Locations and Relevant Anterior Segment Characteristics. Invest Ophthalmol Vis Sci. 2016;57(14):6389-6395.

6. Liu YC, Wilkins M, Kim T, Malyugin B, Mehta JS. Cataracts. Lancet 2017;390:600-12

7. Yanoff M. Indication for lens surgery and different techniques. In: Yanoff M, Duker JS. (editor) Ophthalmology. 2nd ed. St Louis: Mosby; 2004;315-25.

8. Masis Solano M, Lin SC. Cataract, phacoemulsification and intraocular pressure: Is the anterior segment anatomy the missing piece of the puzzle? Prog Retin Eye Res. 2018;64:77-83.

9. Lee W, Bae HW, Kim CY, Seong GJ. The change of anterior segment parameters after cataract surgery in normal-tension glaucoma. Int J Ophthalmol. 2017;10(8):1239-1245.

10. Rękas M, Barchan-Kucia K, Konopiðska J, Mariak Z, Darnowski T. Analy sis and Modeling of Anatomical Changes of the Anterior Segment of the Eye After Cataract Surgery With Consideration of Different Phenotypes of Eye Structure. Curr Eye Res. 2015;40(10):1018-27.

11. Sengupta $S$, Venkatesh R, Krishnamurthy $P$, Nath $M$, Mashruwala $A$, Ramulu PY, et al. Intraocular Pressure Reduction after Phacoemulsification versus Manual Small-Incision Cataract Surgery: A Randomized Controlled Trial. Ophthalmology. 2016;123(8):1695-1703.

12. Beato JN, Reis D, Esteves-Leandro J, Falcão M, Rosas V, Carneiro Â, Falcão Reis F. Intraocular Pressure and Anterior Segment Morphometry Changes after Uneventful Phacoemulsification in Type 2 Diabetic and Nondiabetic Patients. J Ophthalmol. 2019;2019:9390586.

13. Slabaugh MA, Bojikian KD, Moore DB, Chen PP. The Effect of Phacoemulsification on Intraocular Pressure in Medically Controlled Open-Angle Glaucoma Patients. Am J Ophthalmol. 2014;157:26-31.
14. Cetinkaya S, Dadaci Z, Yener HI, Acir NO, Cetinkaya YF, Saglam F. The Effect of Phacoemulsification Surgery on Intraocular Pressure and Anterior Segment Anatomy of the Patients with Cataract and Ocular Hypertension. Indian J Ophthalmol. 2015;63:743-45.

15. Mansberger SL, Gordon MO, Jampel H, Bhorade A, Brandt JD, Wilson B, et al. Reduction in Intraocular Pressure After Cataract Extraction: The Ocular Hypertension Treatment Study. Ophthalmology. 2012;119:1826-31.

16. Huang G, Gonzalez E, Peng PH, Lee R, Leeungurasatien T, He M, et al. Anterior Chamber Depth, Iridocorneal Angle Width and Intraocular Pressure Changes After Phacoemulsification: Narrow vs Open Iridocorneal Angles.Arch Ophthalmol. 2011;129:1283-90.

17. Huang G, Gonzalez E, Lee R, Chen YC, He M, Lin SC. Association of Biometric Factors with Anterior Chamber Angle Widening and Intraocular Pressure Reduction After Uneventful Phacoemulsification for Cataract. J Cataract Refract Surg. 2012;38:108-16.

18. Yang HS, Lee J, Choi S. Ocular Biometric Parameters Associated with Intraocular Pressure Reduction After Cataract Surgery in Normal Eyes. Am J Ophthalmol. 2013;156:89-94.

19. Shrivastava A, Singh K. The effect of cataract extraction on intraocular pressure. Curr Opin Ophthalmol. 2010;21:118-22.

20. Ucakhan OO, Ozkan M, Kanpolat A. Anterior Chamber Parameters Measured by the Pentacam CES after Uneventful Phacoemulsification in Normotensive Eyes. Acta Ophthalmol. 2009;87:544-48.

21. Issa SA, Pacheco J, Mahmood U, Nolan J, Beatty S. A Novel Index for Predicting Intraocular Pressure Reduction Following Cataract Surgery. Br J Ophthalmol. 2005:89:543-46.

22. Yang HS, Lee J, Choi S. Ocular Biometric Parameters Associated with Intraocular Pressure Reduction After Cataract Surgery in Normal Eyes. Am J Ophthalmol. 2013;156:89-94.

23. Kashiwagi K, Kashiwagi F, Tsukahara S. Effects of Small-Incision Phacoemulsification and Intraocular Lens Implantation on Anterior Chamber Depth and Intraocular Pressure. J Glaucoma. 2006;15:103-09.

24. Chen M, Hu H, He W, Lu Y, Zhu X. Observation of Anterior Chamber Volume After Cataract Surgery with Swept-Source Optical Coherence Tomography. Int Ophthalmol. 2019;39:1837-44.

25. Lee RY, Kasuga T, Cui QN, Huang G, Wang SY, Lin SC. Ethnic Differences in Intraocular Pressure Reduction and Changes in Anterior Segment Biometric Parameters Following Cataract Surgery by Phacoemulsification. Clin Exp Ophthalmol. 2013;41:442-49.

26. Altan C, Bayraktar S, Altan T, Eren H, Yilmaz OF. Anterior Chamber Depth, Iridocorneal Angle Width, and Intraocular Pressure Changes After Uneventful Phacoemulsification in Eyes without Glaucoma and with Open Iridocorneal Angles. J Cataract Refract Surg. 2004;30: 832-38. 